\title{
Quantitative neuroimaging and the prediction of rehabilitation outcome following traumatic brain injury
}

\author{
Erin D. Bigler ${ }^{1,2 *}$ and Elisabeth A. Wilde ${ }^{3}$ \\ Department of Psychology and Neuroscience Center, Brigham Young University, Provo, UT, USA \\ 2 Department of Psychiatry, University of Utah, Salt Lake City, UT, USA \\ ${ }^{3}$ Cognitive Neuroscience Laboratory, Departments of Physical Medicine and Rehabilitation, Neurology, and Radiology, Baylor College of Medicine, Houston, TX, USA \\ ${ }^{*}$ Correspondence: erin_bigler@byu.edu
}

\section{A commentary on}

Regional brain morphometry predicts memory rehabilitation outcome after traumatic brain injury.

by Strangman, G. E., O’Neil-Pirozzi, T. M., Supelana, C., Goldstein, R., Katz, D. I., and Glenn, M. B. (2010). Front. Hum. Neurosci. 4:182. doi:10.3389/fnhum.2010.00182.

Diverse motor, sensory, cognitive, and emotional disabilities may be the aftermath of any brain injury (Svestkova et al., 2010). While some systematic interventions exist to treat TBI-related disabilities, there are only a limited number of evidence-based therapies to treat cognitive impairments, which some have argued are the most prevalent of TBI disabilities (Cicerone et al., 2005; Stuss et al., 2008). Potential reasons for the limited number of therapeutic options in treating cognitive deficits following TBI are numerous, but heterogeneity of the injury is at the top of the list (Skandsen et al., 2010). Each brain injury produces a unique set of pathologies not only in the underlying pathophysiology of the injury itself, but also the injury occurs to a brain with its own unique, and individualized organization. Admittedly, there are some universalities that apply to overall brain organization such as general cognitive functions of temporofrontal areas involved in language processing and the medial temporal lobe in memory, but substantial individual differences exist in how even these general regions may be linked to one another and how their underlying circuitry relates to function (Brown et al., 2010). Because of these individual differences, sampling one type of lesion or region of damage in TBI will likely provide limited understanding of the relationship between brain injury, underlying brain pathology, and rehabilitation outcome especially when TBI produces a complex set of diffuse and focal injuries, where multiple brain regions may exhibit atrophic changes (Warner et al., 2010a,b).

Past cognitive rehabilitation TBI research often used neuroimaging findings to define a location of injury or the amount of structural damage, frequently as a singular measure of regional, or whole brain atrophy. All quantitative neuroimaging analysis methods of the past, however required timeconsuming operator-controlled methods that had to be done by hand. Having lesion metrics that not only assess the diffuseness of injury but also location and/or the degree of where atrophic changes have occurred provides the rehabilitation clinician with additional information that may be useful in predicting outcome or even guiding therapies, because it taps the multifaceted manner in which the brain may be injured. However, as already stated, each brain has its own unique organization which means that singular measures such as just lesion volume, localized, or whole brain measures of atrophy fall short of capturing the complexity of the injury, but that is all that could reasonably be done with past quantitative neuroimaging methods. Furthermore, because any quantification in the past required time-consuming methods of analysis, it was not practical for the rehabilitation clinician to even use such information because it could never be reliably generated in a timely fashion. Fortunately, advances in volumetric measurement have become more automated, and as shown by Strangman et al. (2010) in this issue of Frontiers of Human Neuroscience using FreeSurfer quantification techniques, numerous brain regions from conventional T1-weighted volume-acquisition MRI studies can be calculated in the TBI patient needing rehabilitation intervention. Strangman et al. capitalize on this technology and demonstrate that in TBI of all severities, the key to understanding memory outcome following internal memory training strategies is by performing multiple brain morphology measurements (i.e., hippocampal, cingulate, and prefrontal cortical). A singular metric insufficiently captures the complexity of pathological changes in TBI, but multiple metrics do. The internal memory strategies method combined with neuroimaging findings may provide a neurobiological rationale for how to treat memory deficits following TBI (O’Neil-Pirozzi et al., 2010).

Strangman et al. (2010) discuss the implications of their findings with a view toward the future. Evaluating the patency of regions of interest that contribute to memory networks before embarking on cognitive rehabilitation treatment programs may have major implications for TBI rehabilitation. Figure 3 in Strangman et al. shows that if reduced hippocampal and posterior dorsal cingulate volumes are present in TBI patients, then memory deficits are likely to remain substantially impaired, even after the structured memory therapies that were applied in this study.

There are other more futuristic implications of this research. Diffusion tensor imaging (DTI) provides methods to examine the health of white matter connections between regions of interest, and may be particularly sensitive in assessing affected pathways in TBI (Niogi and Mukherjee, 2010). Functional MRI studies are also showing the utility of fMRI activation patterns in assessing the effects of brain injury (Laatsch, 2007). Combining fMRI and DTI with the type of morphometric structural analysis done by Strangman et al. could be important in assessing not only the effectiveness of a treatment regimen, but in determining which rehabilitation treatment modalities are likely to be most useful to an individual patient. Strangman et al. discuss some of these potentialities and this line of research 
will hopefully lead to new innovative findings that blends neuroimaging technology with treatment.

As Strangman et al. point out, FreeSurfer is freely available and open for use by any radiology department or neuroimaging center, with the image analysis largely automated, allowing for the potential use of such software in aspects of clinical decisionmaking. DTI and fMRI analysis programs are also becoming more available, accessible, automated and inexpensive, and combinations of these technologies may be quite fruitful in the future. As always, there are complexities in imaging-behavior relationships that require careful consideration and additional research. For example, the Strangman et al. study utilized participants with chronic TBI, at an interval where both morphometric changes and cognitive recovery have been considered to be relatively stable. However, the relationship between brain morphometry and the cognitive capacity to benefit from rehabilitation strategies may be much more dynamic and complex in the earlier phases of recovery. In the first few months post-injury, an interval where clinical decision-making is being applied for many individuals with TBI, the trajectories of rapid cognitive recovery and incomplete degenerative tissue change may be progressing in opposing directions and to differing degrees. Clearly, much still needs to be understood about this relationship in order for these techniques to be widely utilized as predictive tools in a clinical setting. Additionally, and as mentioned in the study, automated programs for volumetric measurement such as FreeSurfer may have some accuracy limita- tions in regions of the brain that are difficult to model (e.g., medial temporal areas) or that contain very large lesions or types of pathology that the software cannot accurately distinguish. Furthermore, the general artifacts and limitations of MRI (e.g., metal artifact, motion, etc.) may limit its use in some individuals with TBI, particularly in early subacute stages. Finally, determination of TBI-related cortical change requires an appropriate normative comparison given the dynamic developmental changes that occur throughout the lifespan, particularly in children, adolescents, and the elderly. Despite these caveats, advanced quantitative imaging such as the techniques applied in the Strangman et al. study will likely advance our understanding of the effects of brain injury and how best to conduct rehabilitation therapies in the TBI patient.

\section{REFERENCES}

Brown, G. G., Mathalon, D. H., Stern, H., Ford, J., Mueller, B., Greve, D. N., McCarthy, G., Voyvodic, J., Glover, G., Diaz, M., Yetter, E., Ozyurt, I. B., Jorgensen, K. W., Wible, C. G., Turner, J, A., Thompson, W. K., and Potkin, S. G. (2010). Function Biomedical Informatics Research Network. Multisite reliability of cognitive BOLD data. Neuroimage. PMID: 20932915. [Epub ahead of print].

Cicerone, K. D., Dahlberg, C., Malec, J. F., Langenbahn, D. M., Felicetti, T., Kneipp, S., Ellmo, W., Kalmar, K. Giacino, J. T., Harley, J. P., Laatsch, L., Morse, P. A., and Catanese,J. (2005). Evidence-based cognitive rehabilitation: updated review of the literature from 1998 through 2002. Arch. Phys. Med. Rehabil. 86, 1681-1692.

Laatsch, L. (2007). The use of functional MRI in traumatic brain injury diagnosis and treatment. Phys. Med. Rehabil. Clin. N. Am. 18, 69-85.

Niogi, S. N., and Mukherjee, P. (2010). Diffusion tensor imaging of mild traumatic brain injury. J. Head Trauma Rehabil. 25, 241-255.

O’Neil-Pirozzi, T. M., Strangman, G. E., Goldstein, R., Katz, D. I., Savage, C. R., Kelkar, K., Supelana, C., Burke, D., Rauch, S. L., and Glenn, M. B. (2010). A con- trolled treatment study of internal memory strategies (I-MEMS) following traumatic brain injury. J. Head Trauma Rehabil. 25, 43-51.

Skandsen, T., Kvistad, K. A., Solheim, O., Strand, I. H. Folvik, M., and Vik, A. (2010). Prevalence and impact of diffuse axonal injury in patients with moderate and severe head injury: a cohort study of early magnetic resonance imaging findings and 1-year outcome. J. Neurosurg. 113, 556-563.

Strangman, G. E., O’Neil-Pirozzi, T. M., Supelana, C., Goldstein, R., Katz, D., and Glenn, M. B. (2010) Regional brain morphometry predicts memory rehabilitation outcome after traumatic brain injury. Front. Hum. Neurosci. 4:182. doi: 10.3389/ fnhum.2010.00182.

Stuss, D. T., Winocur, G., and Robertson, I. H. (2008). Cognitive Neurorehabilitation, 2nd Edn. New York: Cambridge University Press.

Svestkova, O., Angerova, Y., Sladkova, P., Bickenbach, J. E., and Raggi, A. (2010). Functioning and disability in traumatic brain injury. Disabil. Rehabil. 32(Suppl. 1), S68-S77.

Warner, M. A., Marquez de la Plata, C., Spence, J., Wang, J. Y., Harper, C., Moore, C., Devous, M., and DiazArrastia, R. (2010a). Assessing spatial relationships between axonal integrity, regional brain volumes, and neuropsychological outcomes after traumatic axonal injury. J. Neurotrauma. doi: 10.1089/neu. 2010. 1429. [Epub ahead of print].

Warner, M.A., Youn, T.S., Davis, T., Chandra, A., Marquez de la Plata, C., Moore, C., Harper, C., Madden, C. J., Spence, J., McColl, R., Devous, M., King, R. D., and Diaz-Arrastia, R. (2010b). Regionally selective atrophy after traumatic axonal injury. Arch Neurol. 67, 1336-1344.

Received: 27 October 2010; accepted: 30 November 2010 published online: 23 December 2010.

Citation: Bigler ED and Wilde EA (2010) Quantitative neuroimaging and the prediction of rehabilitation outcome following traumatic brain injury. Front. Hum. Neurosci. 4:228. doi: 10.3389/fnhum.2010.00228

Copyright ( $\odot 2010$ Bigler and Wilde. This is an open-access article subject to an exclusive license agreement between the authors and the Frontiers Research Foundation, which permits unrestricted use, distribution, and reproduction in any medium, provided the original authors and source are credited. 